\title{
Lactobacillus rhamnosus alleviates intestinal barrier dysfunction in part by increasing expression of zonula occludens-1 and myosin light-chain kinase in vivo
}

\author{
E. Miyauchi, ${ }^{* 1}$ H. Morita, $†$ and S. Tanabe* \\ *Graduate School of Biosphere Science, Hiroshima University, 1-4-4 Kagamiyama, Higashi-hiroshima, Hiroshima 739-8528, Japan \\ †School of Veterinary Medicine, Azabu University, 1-17-71 Fuchinobe, Sagamihara, Kanagawa 229-8501, Japan
}

\begin{abstract}
The effects of lactobacilli on impaired intestinal barrier function and paracellular permeability were evaluated in human epithelial Caco-2 cells treated with tumor necrosis factor- $\alpha$ and in mice with colitis induced by dextran sodium sulfate (DSS). Filter-grown Caco-2 monolayers were used as the intestinal epithelial model. Among the 4 lactobacilli studied, Lactobacillus rhamnosus OLL2838 most effectively suppressed barrier impairment and increased IL-8 secretion induced by tumor necrosis factor- $\alpha$ in Caco-2 cells; however, the conditioned medium from OLL2838 did not show any effect on barrier functions. The in vivo effects of OLL2838 on intestinal epithelial barrier function and colonic inflammation were assessed in DSS-induced colitis of BALB/c mice. Oral treatment with both live and heat-killed OLL2838 suppressed weight loss and recovered colon length. Additionally, barrier function was restored by the administration of live and heat-killed OLL2838 to the DSS-treated animals, which conferred protection against the increase in mucosal permeability associated with DSS-induced colitis. This may at least partially be because of the increased expression of zonula occludens-1 (4.8-fold) and myosin light-chain kinase (3.1-fold) in intestinal epithelial cells isolated from mice of the heat-killed OLL2838 group. Therefore, $L$. rhamnosus OLL2838 would be useful in the treatment of gastrointestinal diseases such as inflammatory bowel disease.
\end{abstract}

Key words: Lactobacillus rhamnosus, colitis, intestinal barrier, myosin light-chain kinase

\section{INTRODUCTION}

Inflammatory bowel diseases (IBD) such as ulcerative colitis and Crohn's disease are chronic inflamma-

Received September 10, 2008.

Accepted February 10, 2009.

${ }^{1}$ Corresponding author: emiyauchi@hiroshima-u.ac.jp tory disorders of the gastrointestinal tract. Recently, there have been substantial advances in understanding the molecular pathogenesis of IBD (Xavier and Podolsky, 2007). One of the mechanisms involved is intestinal barrier dysfunction and the resulting responses to commensal bacteria (microbiota). Although the host immune system is usually tolerant to most commensal bacteria, primary dysregulation of the mucosal immune system leads to excessive immunological responses, even against normal microbiota (Strober et al., 2007).

The host immune system is substantially separated from the microbiota by a layer of epithelial cells whose integrity is maintained by intercellular junctional complexes; for example, tight junctions (TJ). The TJ contains several proteins of which zonula occludens-1 (ZO-1) was the first to be identified. The ZO-1 protein binds to transmembrane proteins such as claudins and occludin and links them to cytoskeletal actin. Marked downregulation of junction proteins has been observed in actively inflamed IBD tissue (Zeissig et al., 2007). This leads to increased TJ permeability and decreased transepithelial resistance (TER) in mice (Poritz et al., 2007). The increased permeability in IBD patients is related to their disease activity and is predictive of relapse after pharmacological and surgical relief from inflammation (Meddings, 2008).

Inflammatory cytokines such as tumor necrosis factor- $\alpha$ (TNF- $\alpha$ ) induce intestinal barrier impairment both in vitro and in vivo (Li et al., 2008; Mazzon and Cuzzocrea, 2008). The TNF- $\alpha$-induced decrease in TER and increase in permeability are associated with downregulation of ZO-1 protein expression and changes in the junctional localization in vitro (Ma et al., 2004). Ma et al. (2005) have also demonstrated that the TNF- $\alpha$-induced TER decrease is partly mediated by an increase in expression of myosin light-chain kinase (MLCK) protein and its activation in vitro.

Increasing evidence suggests that some probiotic and commensal bacteria ameliorate inflammation in IBD patients (Gionchetti et al., 2007; Soo et al., 2008) and in vitro. This effect is attributed to the following mechanisms: inhibition of TNF- $\alpha$ production (Lin et 
al., 2008), enhancement of antiinflammatory cytokines such as IL-10 (Smits et al., 2005) and peroxisome proliferator-activated receptor gamma (Kelly et al., 2004), and antimicrobial substance induction (Schlee et al., 2008). Furthermore, certain probiotic and commensal bacteria suppress intestinal barrier impairment in vitro (Resta-Lenert and Barrett, 2006; Miyauchi et al., 2008). However, few studies have demonstrated in vivo that these kinds of bacteria can ameliorate intestinal inflammation by recovering intestinal barrier function.

If it can be demonstrated that lactobacilli help recover intestinal barrier function, then even children could benefit by consuming lactobacilli in affordable food products such as yogurt. Here, we compared the effects of 4 lactobacilli that are frequently used for fermented milk processing on barrier function in vitro. The strain exerting the most potent activity in vitro was evaluated for amelioration of intestinal inflammation by recovering the intestinal barrier function in mice with dextran sodium sulfate (DSS)-induced colitis.

\section{MATERIALS AND METHODS}

\section{Chemicals and Media}

Dulbecco's modified Eagle's medium (DMEM), nonessential amino acids, penicillin, streptomycin, gentamycin, and Hanks' balanced salt solution were all obtained from Invitrogen Corp. (Carlsbad, CA). Fetal bovine serum, Transwell cell culture chambers $(0.4-\mu \mathrm{m}$ pore size, 12-mm diameter), Millicell-ERS instrument with $\mathrm{Ag} / \mathrm{AgCl}$ electrodes, and $\mathrm{TNF}-\alpha$ were purchased from ICN Biomedicals Inc. (Costa Mesa, CA), Corning Costar (Cambridge, MA), Millipore (Bedford, MA), and Wako Pure Chemical Industries Ltd. (Osaka, Japan), respectively. All other chemicals were of reagent grade.

\section{Caco-2 Cells}

Caco-2 cells were purchased from the American Type Culture Collection (Rockville, MD). These cells were between 35 and 45 passages. The growth medium contained DMEM with $10 \%$ fetal bovine serum, $1 \%$ nonessential amino acids, and antibiotics (100 units/ $\mathrm{mL}$ penicillin, $100 \mu \mathrm{g} / \mathrm{mL}$ streptomycin, and $50 \mu \mathrm{g} / \mathrm{mL}$ gentamycin). The cells were cultured at $37^{\circ} \mathrm{C}$ under a humidified $5 \% \mathrm{CO}_{2}$ atmosphere in $75-\mathrm{cm}^{2}$ tissue culture flasks to approximately $80 \%$ confluence and seeded into a 12-well Transwell cell culture chamber (density, $5 \times$ $10^{4}$ cells $/ \mathrm{cm}^{2}$ ). After $14 \mathrm{~d}$ of culture, TER was measured using a Millicell-ERS instrument. Caco-2 cell monolayers were used when their TER values were greater than $300 \Omega / \mathrm{cm}^{2}$. Wells were placed in cluster plates with an outside medium (basolateral side, $1.5 \mathrm{~mL}$ ) and an inside medium (apical side, $0.5 \mathrm{~mL}$ ). The monolayers were replenished with fresh medium every $24 \mathrm{~h}$.

\section{Evaluation of Barrier Function In Vitro}

Lactobacillus delbrueckii ssp. bulgaricus no. 3, Lactobacillus casei no. 9, Lactobacillus gasseri no. 10, and Lactobacillus rhamnosus OLL2838 were obtained from Meiji Dairies Corporation (Kanagawa, Japan). Strains no. 3 and no. 9 were isolated from dairy products and raw milk, respectively, whereas no. 10 and OLL2838 were obtained from human feces. They were cultured in de Man, Rogosa, and Sharpe broth (Becton Dickinson, Sparks, MD) and incubated under anaerobic conditions by using AnaeroPack (Mitsubishi Gas Chemical, Tokyo, Japan) at $37^{\circ} \mathrm{C}$ for $18 \mathrm{~h}$. The culture suspensions were washed with distilled water. Subsequently, the lactobacilli $\left(10^{5} \mathrm{cfu} /\right.$ well $)$ were added to the apical side of the Caco-2 cell monolayers. When adding the conditioned medium, $2 \times 10^{5} \mathrm{cfu} / \mathrm{mL}$ of the lactobacilli were incubated in DMEM for $48 \mathrm{~h}$; then, the lactobacilli were removed using a $0.22-\mu \mathrm{m}$ filter and the removal was verified by plating serial dilutions. One hour later, the cells were treated with TNF- $\alpha(100 \mathrm{ng} / \mathrm{mL})$ on the basolateral side of the monolayers and cultured for $48 \mathrm{~h}$. After the 48-h incubation, the TER value was measured to assess epithelial barrier function. The basolateral medium was collected, and the IL-8 concentration of the supernatant was measured using a DuoSet sandwich ELISA (R\&D Systems, Abingdon, UK; Miyauchi et al., 2008). After removing the supernatant, the Caco-2 cells were scraped and used for SDS-PAGE and Western blotting, as described below. All experiments were performed in triplicate.

\section{SDS-PAGE and Western Blotting}

Caco-2 cell monolayers and mice colonic epithelial cells were washed thrice with ice-cold PBS ( $\mathrm{pH} 7.4$ ) and lysed in lysis buffer $(25 \mathrm{~m} M$ TrisHCl, $150 \mathrm{mM}$ $\mathrm{NaCl}, 1 \%$ Nonidet P-40, $1 \%$ sodium deoxycholate, and $1 \%$ SDS; pH 7.4). The cell suspensions were sonicated for 30 min using a sonicator (USK-3, As One Corp., Osaka, Japan) and centrifuged at 14,000 $\times g$ for $30 \mathrm{~min}$ at $4^{\circ} \mathrm{C}$ to yield a clear lysate. The proteins were then separated using a $7.5 \%$ gel and transferred onto blotting membranes (Immobilon $\mathrm{P}$, polyvinylidene fluoride, Millipore). After overnight blocking with PBS (pH 7.4) supplemented with $0.1 \%$ Tween-20 and $1 \%$ BSA, the blots were incubated with primary (rabbit anti-ZO-1 or mouse anti-MLCK) and secondary antibodies (horseradish peroxidase-conjugated anti-rabbit or anti-mouse IgG, as appropriate) for $1 \mathrm{~h}$ each at room temperature. 
Anti-ZO-1 antibody was obtained from Zymed Labs (San Francisco, CA); anti-MLCK antibody and horseradish peroxidase-conjugated anti-mouse IgG were obtained from Sigma-Aldrich (St Louis, MO); and horseradish peroxidase-conjugated anti-rabbit IgG was obtained from Kirkegaard \& Perry Lab (Baltimore, MD). To confirm equal loading, the blots were reprobed with rat anti- $\alpha$-tubulin antibody (Chemicon International, CA). The proteins were visualized using chemiluminescence (GE Healthcare, Buckinghamshire, UK) and exposed to x-ray film (Fujifilm, Kanagawa, Japan). Band densities were read using Scion Image Software (Scion, Frederick, $\mathrm{MD})$, and standardized with $\alpha$-tubulin.

\section{Animal Studies}

BALB/c mice (female, 6-wk-old) were purchased from Charles River Japan (Kanagawa, Japan) and housed in an air-conditioned room under a 12-h light-dark cycle. The mice were provided ad libitum access to tap water and a standard diet (MF; Oriental Yeast, Tokyo, Japan). The experimental protocols were approved by the Animal Care Committee, Graduate School of Biosphere Science, Hiroshima University, Japan. Acute colitis was induced in mice by adding $3.5 \%$ (wt/vol) DSS (molecular weight, 36,000-50,000; MP Biomedicals, Aurora, $\mathrm{OH})$ to their drinking water for $5 \mathrm{~d}$.

Animal studies were conducted using the species with the highest barrier-recovering activity in vitro among the 4 species tested. Animals were randomly separated into 4 groups $(\mathrm{n}=3$ or $\mathrm{n}=4)$. Group 1 (normal group) mice were treated orally with PBS from d 1 to 3 ; group 2 (control group) mice received drinking water with $3.5 \%$ DSS and were orally administered PBS. Groups 3 and 4 also received drinking water with $3.5 \%$ DSS and $24 \mathrm{~h}$ after the start of DSS administration, mice were orally administered $10^{7} \mathrm{cfu} / \mathrm{d}$ of live or heat-killed lactobacilli for 3 consecutive days. The heat-killed bacteria were prepared by heating to $75^{\circ} \mathrm{C}$ for $1 \mathrm{~h}$ followed by lyophilization. The animals' BW were measured daily. The mice were killed under anesthesia with diethyl ether on d 5. The entire colon was removed from the cecum to the anus and its length was measured as a marker of inflammation.

\section{Tissue Myeloperoxidase Activity}

Myeloperoxidase activity was measured in the distal colon segments. Samples were rinsed with cold PBS, blot dried, and immediately frozen in liquid nitrogen and stored at $-80^{\circ} \mathrm{C}$ until assay for activity by the o-dianisidine method (Gebska et al., 2005; Krieglstein et al., 2007). For the assay, tissue samples were thawed and weighed, suspended (10\% wt/vol) in $50 \mathrm{mM}$ potassium phosphate buffer ( $\mathrm{pH}$ 6.0) containing $0.5 \%$ hexadecyltrimethylammonium bromide, and then homogenized. The homogenate was centrifuged at 14,000 $\times g$ for $10 \mathrm{~min}$ at $4^{\circ} \mathrm{C}$. The reaction was initiated by mixing and incubating the supernatant $(50 \mu \mathrm{L})$ at $20^{\circ} \mathrm{C}$ for 10 min with a solution composed of $1,420 \mu \mathrm{L}$ of $50 \mathrm{~m} M$ potassium phosphate, $15 \mu \mathrm{L}$ of $20 \mathrm{mg} / \mathrm{mL} \mathrm{o-}$ dianisidine dihydrochloride, and $15 \mu \mathrm{L}$ of $20 \mathrm{~m} M$ hydrogen peroxide. After $5 \mathrm{~min}$, the reaction was terminated by adding $10 \mu \mathrm{L}$ of $2 \%$ sodium azide. The change in absorbance was read at $460 \mathrm{~nm}$ in a spectrophotometer. Myeloperoxidase activity was expressed as the amount of enzyme necessary to produce a change in absorbance of $1.0 \mathrm{unit} / \mathrm{min}$ per gram of tissue (wet weight).

\section{In Vivo Intestinal Permeability}

Intestinal permeability was examined by the method of Cario et al. (2007) with some modifications. Briefly, on d 5 , mice were gavaged with $0.6 \mathrm{mg} / \mathrm{g}$ of $\mathrm{BW}$ of fluorescein isothiocyanate (FITC)-labeled dextran (mol wt, 4,000). The amount of FITC-labeled dextran in the blood was determined $4 \mathrm{~h}$ later by measuring fluorescence intensity with a spectrophotofluorometer (Nihon Bunko, FP-750, Tokyo, Japan) at excitation and emission wavelengths of $495 \mathrm{~nm}$ and $520 \mathrm{~nm}$, respectively.

\section{Isolation of Mouse Colonic Epithelial Cells}

On d 5, the mice were killed under anesthesia with diethyl ether, and the distal colon $(5 \mathrm{~cm}$ before the anus) was removed. Colonic epithelial cells were partially purified by the method of Lee et al. (2006) with some modifications. Briefly, the whole colon was washed with PBS, minced, washed thrice in PBS containing 1 $\mathrm{m} M$ dithiothreitol, and incubated in Hanks' balanced salt solution containing $5 \mathrm{~m} M$ EDTA and $0.1 \mathrm{~m} M$ dithiothreitol for $1 \mathrm{~h}$ at $37^{\circ} \mathrm{C}$. The sample was shaken vigorously to detach epithelial cells and crypts, which were then collected from the supernatant after allowing the large tissue pieces to settle to the bottom of the tube. The collected cells and crypts were centrifuged at $800 \times g$ for $5 \mathrm{~min}$ and washed thrice in PBS. The pellet was subjected to SDS-PAGE and analyzed by Western blotting with anti-ZO-1 or anti-MLCK, as described earlier.

\section{Data Analyses}

All data are expressed as the means \pm standard error. Statistical analysis was performed using one-way ANOVA followed by Tukey's post-hoc test. 


\section{RESULTS}

\section{L. rhamnosus OLL2838 Suppressed TNF-a-Induced Barrier Impairment in Caco-2 Cells}

In this study, TJ barrier impairment was induced by TNF- $\alpha$ in the human epithelial Caco-2 cells and evaluated by measuring the TER value. The effects of live $L$. delbrueckii no. 3, L. casei no. 9, L. gasseri no. 10, and L. rhamnosus OLL2838 on TNF- $\alpha$-induced changes in TJ barrier function (TER) and the level of IL-8, one of the major proinflammatory cytokines, were studied. Because these effects were most prominent when the Caco-2 cells were incubated with $10^{5} \mathrm{cfu} /$ well of live lactobacilli, all the experiments were carried out at this bacterial count. Figure 1A shows that TNF- $\alpha$ treatment decreased TER. However, pretreatment with OLL2838 significantly suppressed the TNF- $\alpha$-induced decrease in TER. On the other hand, the effects of $L$. delbrueckii no. 3, L. casei no. 9, and L. gasseri no. 10 were rather weak, with no significant differences between the TER values of the control and these 3 strains. As shown in Figure 1B, TNF- $\alpha$ treatment also induced a remarkable increase in the production of IL- 8 in Caco- 2 cells. The OLL2838 strain significantly suppressed TNF$\alpha$-induced IL- 8 production, and the other lactobacilli suppressed IL-8 production to some extent.

We determined whether the bacterial components or metabolites secreted into the medium were responsible for the observed effect. The conditioned medium from OLL2838 did not show the effects on TER that OLL2838 had on Caco-2 cells (Figure 2A). Thus, nonsecreted components of OLL2838 were considered important for this effect.

To assess the changes in the expression of TJ-associated and TJ-regulating proteins, a Western blot was performed using anti-ZO-1 and anti-MLCK antibodies (Figure 3). To confirm equal loading, the blots were reprobed with anti- $\alpha$-tubulin antibody. Tumor necrosis factor- $\alpha$ drastically decreased ZO-1 expression and increased MLCK expression. However, OLL2838 markedly increased ZO-1 levels and decreased MLCK levels. Therefore, we confirmed that OLL2838 suppressed TJ barrier impairment in the in vitro model.

\section{Effect of Live and Heat-Killed OLL2838 on Mice with DSS-Induced Colitis}

We evaluated whether OLL2838 could ameliorate intestinal inflammation in a mice colitis model. Acute colitis was induced in mice by addition of $3.5 \%$ DSS to their drinking water for $5 \mathrm{~d}$. Mice were given either live or heat-killed OLL2838 orally for 3 consecutive days $24 \mathrm{~h}$ after DSS administration was started (Figure
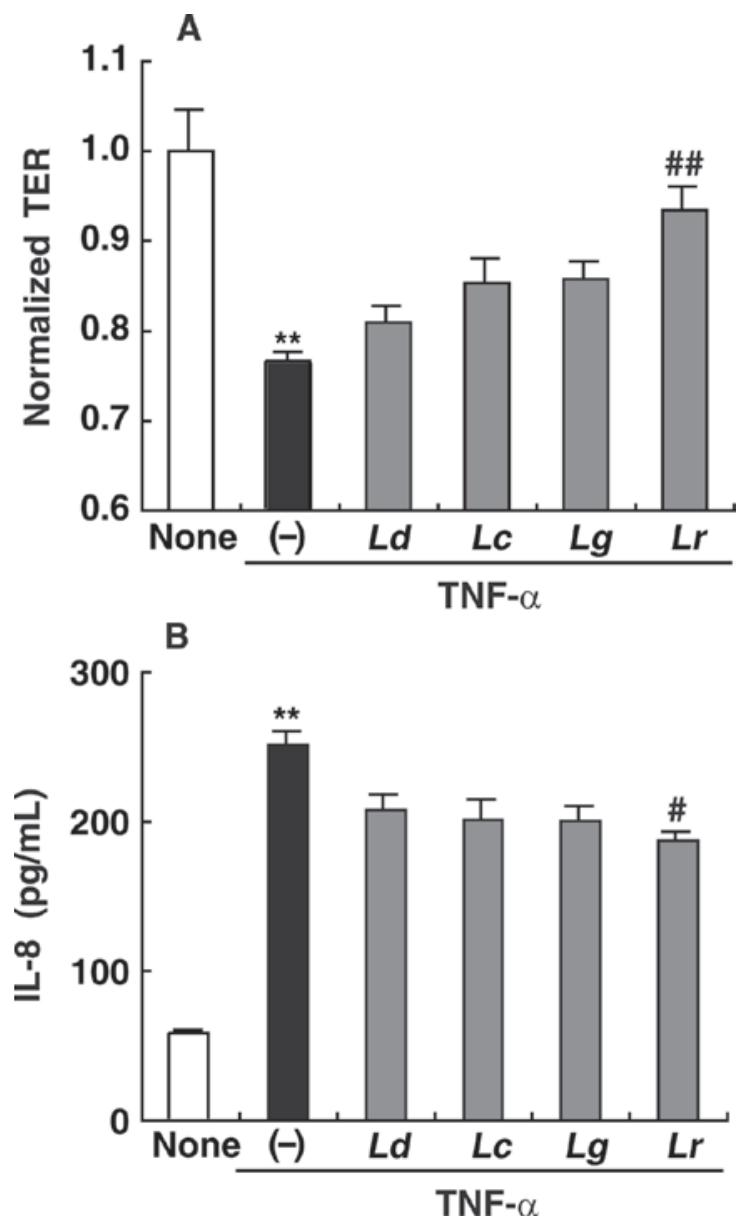

Figure 1. Protective effect of 4 lactobacilli on tumor necrosis factor- $\alpha$ (TNF- $\alpha)$-induced changes in A) transepithelial resistance (TER) and B) IL- 8 secretion by Caco- 2 cells. Caco- 2 cell monolayers were treated with each species $\left(10^{5} \mathrm{cfu} /\right.$ well $)$ or with medium alone (control) for $1 \mathrm{~h}$, and the monolayers were exposed to TNF- $\alpha$. Results are expressed as means \pm standard error $(\mathrm{n}=3)$. ${ }^{*} * P<0.01$ versus controls (none); \#P<0.05 and \#\#P<0.01 versus TNF- $\alpha$ alone $(-)$. $L d=$ Lactobacillus delbrueckii ssp. bulgaricus no. $3 ; L c=$ Lactobacillus casei no. 9; Lg= Lactobacillus gasseri no. 10; Lr = Lactobacillus rhamnosus OLL2838.

4A). Upon DSS administration, the BW of the mice decreased markedly, which was suppressed by supplementation with live or heat-killed OLL2838 (Figure 4B). Moreover, DSS caused a decrease in colon length (Figure 4C and D), an observation also reported by others (Fitzpatrick et al., 2007; Krieglstein et al., 2007; Poritz et al., 2007). However, both live and heat-killed OLL2838 improved these lesions.

Myeloperoxidase activity in the distal colon also increased on DSS administration, suggesting the development of inflammation. Live but not heat-killed OLL2838 decreased colonic myeloperoxidase activity (Figure 5). No changes in myeloperoxidase activity were observed in the proximal colon following DSS and OLL2838 administration. 

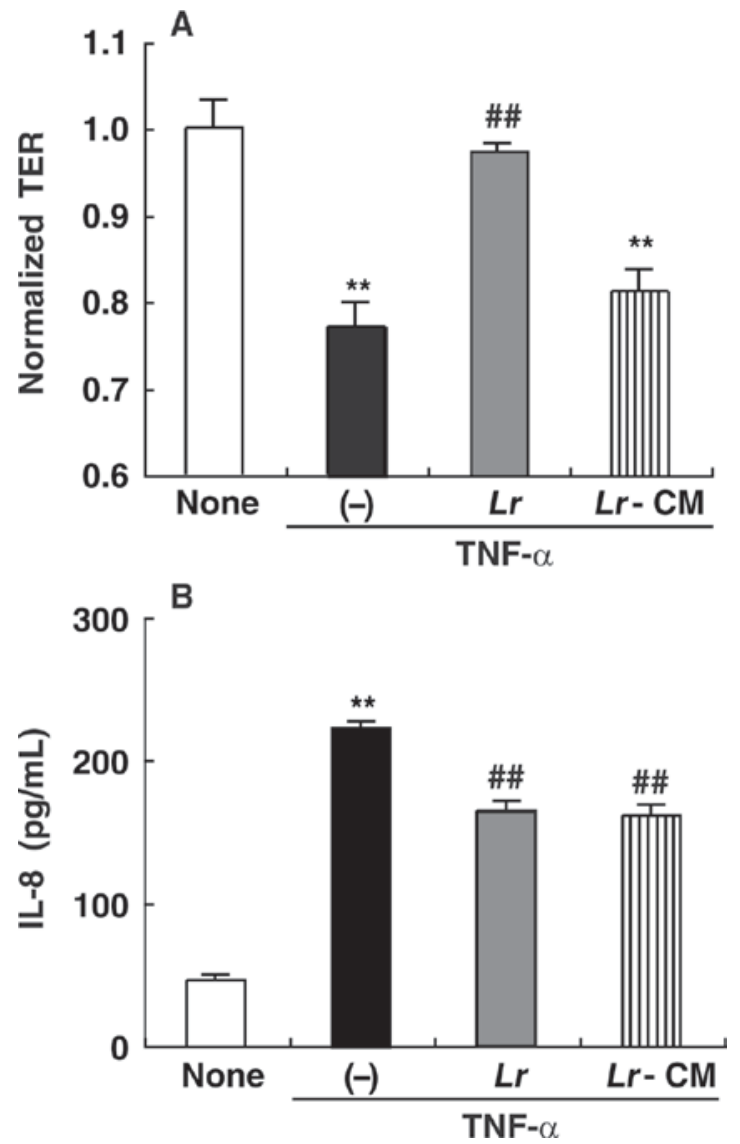

Figure 2. Protective effect of Lactobacillus rhamnosus OLL2838 $(L r)$ and OLL2838-conditioned medium $(L r-\mathrm{CM})$ on tumor necrosis factor- $\alpha$ (TNF- $\alpha)$-induced change in A) transepithelial resistance (TER) and B) IL-8 secretion by Caco-2 cells. Caco-2 cell monolayers were treated with OLL2838 $\left(10^{5} \mathrm{cfu} /\right.$ well $)$, OLL2838-conditioned medium, or medium alone for $1 \mathrm{~h}$ and exposed to TNF- $\alpha$. Results are expressed as means \pm standard error $(\mathrm{n}=3)$. ${ }^{* *} P<0.01$ versus controls (none); \#\#P<0.01 versus TNF- $\alpha$ alone $(-)$.

To evaluate mucosal barrier function in these mice, we administered FITC-labeled dextran and measured the serum fluorescence intensity $4 \mathrm{~h}$ later. The serum levels of FITC-labeled dextran were elevated on DSS administration, but supplementation with either live or heat-killed OLL2838 decreased the levels of FITClabeled dextran, which were comparable to that of healthy mice (Figure 6A). Further, as observed in the Caco-2 cells (Figure 3A), both live and heat-killed OLL2838 remarkably recovered the ZO-1 level in the colonic epithelium, which was drastically suppressed by DSS (Figure 6B).

In contrast to the data obtained for the $\mathrm{TNF}-\alpha$-treated Caco-2 cells (Figure 3B), MLCK expression in the colonic epithelium of DSS-induced colitis mice diminished during barrier impairment (Figure 6C). Both live and heat-killed OLL2838 recovered the MLCK level.
A

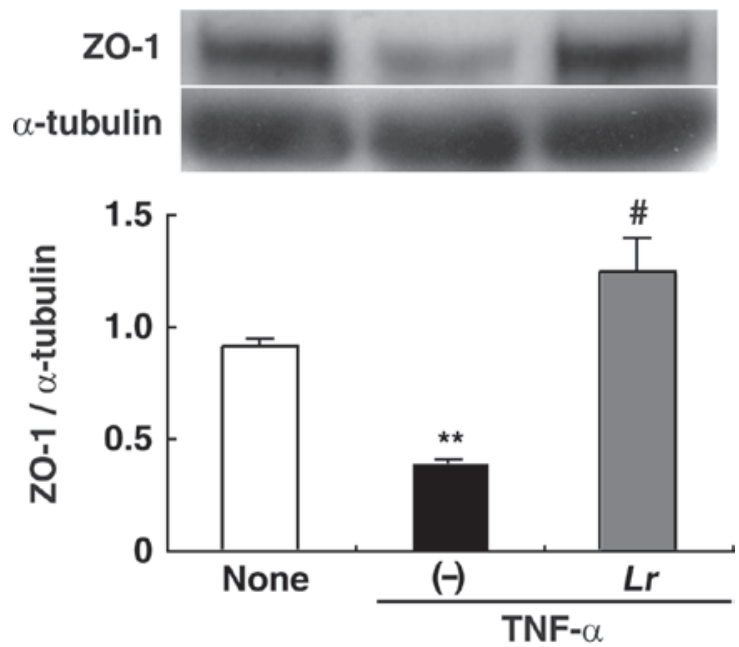

B
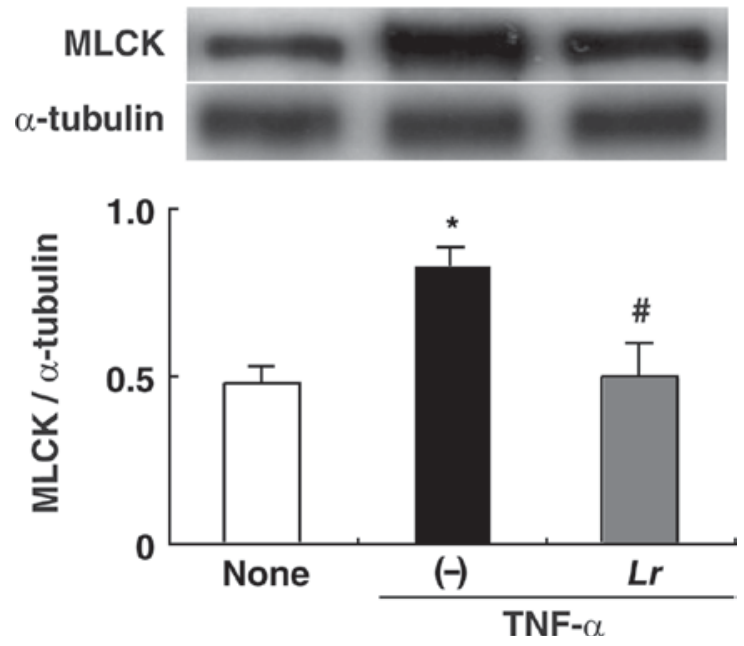

Figure 3. The effect of Lactobacillus rhamnosus OLL2838 on expression of A) zonula occludens-1 (ZO-1) and B) myosin light-chain kinase (MLCK) in Caco-2 cells. Caco-2 cell monolayers were treated with OLL2838 $\left(10^{5} \mathrm{cfu} /\right.$ well $)$ or medium alone for $1 \mathrm{~h}$ and exposed to tumor necrosis factor- $\alpha$ (TNF- $\alpha$ ). The expression of ZO-1, MLCK, and $\alpha$-tubulin was detected by Western blotting of the cellular lysates. Images are representative of 3 independent experiments. Results are expressed as means \pm standard error $(\mathrm{n}=3)$. ${ }^{*} P<0.05$ and ${ }^{*} P<$ 0.01 versus the controls (none); $\# P<0.05$ versus $\mathrm{TNF}-\alpha$ alone $(-)$.

\section{DISCUSSION}

In this study, we demonstrated that OLL2838 is effective for the suppression of TNF- $\alpha$-induced barrier impairment in Caco-2 cells and DSS-induced colitis in mice. Several groups have reported similar effects of $L$. rhamnosus GG in vitro (Zhang et al., 2005; Choi et al., 2008; Seth et al., 2008) and in IBD patients (Zocco et al., 2006). However, we compared the antiinflammatory activity among 4 lactobacilli used in fermented 
A

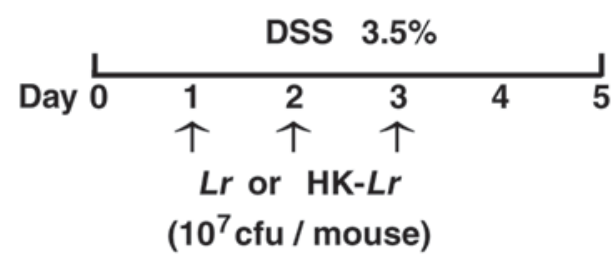

C

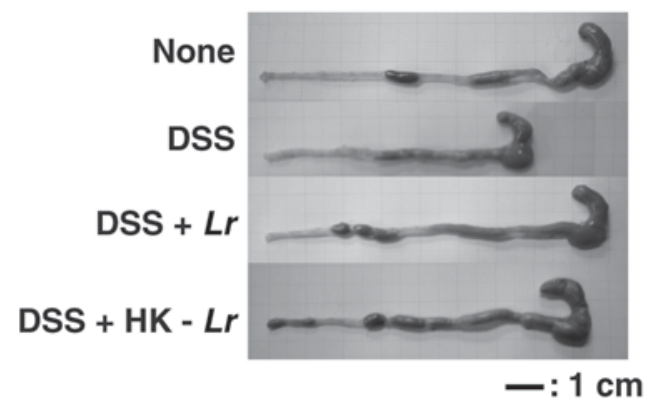

B
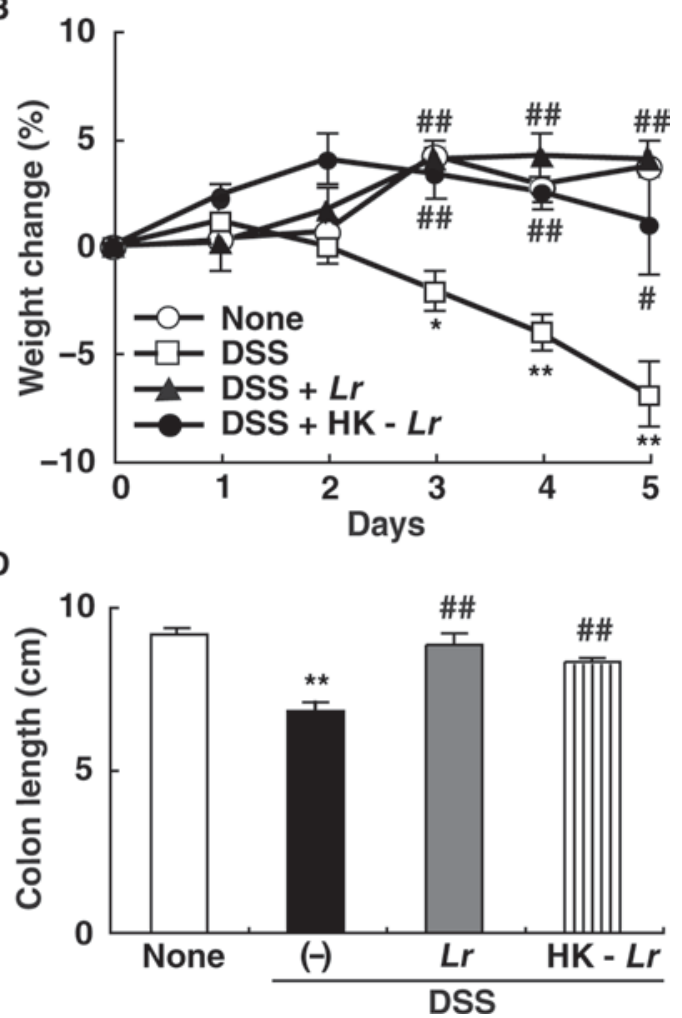

Figure 4. The effect of supplementation of live $(L r)$ or heat-killed (HK- Lr) Lactobacillus rhamnosus OLL2838 on dextran sodium sulfate (DSS)-induced colitis. A) Study design; B) body weight change; C) macroscopic view of the colons on d 5; and D) colon length on d 5. Results are expressed as means \pm standard error $(\mathrm{n}=4)$. ${ }^{*} P<0.05$ and ${ }^{* *} P<0.01$ versus controls (none); \#P<0.05 and \#\#P<0.01 versus DSStreated mice (DSS).

milk food production and demonstrated the barrierrecovering activity of OLL2838 in vivo.

As compounds responsible for this antiinflammatory activity of other strains of L. rhamnosus, soluble protein secreted by L. rhamnosus GG (Seth et al., 2008) and the conditioned medium of $L$. rhamnosus GG (Choi et al., 2008) have been reported. Zhang et al. (2005) reported that both live and dead L. rhamnosus GG inhibited TNF- $\alpha$-induced IL- 8 production in Caco- 2 cells. We also found that both live and heat-killed OLL2838 suppressed DSS-induced colitis, although the antiinflammatory ability of heat-killed OLL2838 was weaker than that of live L. rhamnosus (Figures $4 \mathrm{~B}$ and 5).

The cell wall component in heat-killed OLL2838 is thought to be responsible for the observed effect. We previously reported that the cell wall fraction of the commensal bacteria Enterococcus hirae ameliorated TNF- $\alpha$-induced barrier impairment in Caco-2 cells (Miyauchi et al., 2008). Further, it was demonstrated that $E$. hirae cell wall fractions (e.g., lipoteichoic acid) protected against intestinal impairment by regulating the epithelial TJ via Toll-like receptor (TLR)-2 signaling. Cario et al. (2007) also reported that the synthetic TLR2 ligand $\mathrm{Pam}_{3}$ Cys-Ser-(Lys) ${ }_{4}$ ameliorated the dam- age in Caco-2 cells and in intestinal cells in a mice colitis model. Furthermore, Lee et al. (2006) demonstrated that apical TLR9 signaling in intestinal epithelial cells was important for maintaining colonic homeostasis. Additional studies are required to clarify whether TLR is involved in the antiinflammatory effects of OLL2838 observed in this study.

Dextran sodium sulfate induced the decrease in $\mathrm{ZO}-1$ expression in the mice colonic epithelium (Figure $6 \mathrm{~B}$ ). Because ZO-1 is an integral component of other junctional complexes, including the adherens and gap junctions, its downregulation affects these junctions (Ma et al., 2004). The level of ZO-1 in the epithelium was markedly recovered by live and heat-killed OLL2838 (Figure 6B). Thus, this increased ZO-1 expression, at least in part, leads to the enforcement of TJ barrier function and the reduced permeability of FITC-labeled dextran (Figure 6A).

As shown in Figure 3, TNF- $\alpha$ induced the increase in MLCK expression and paracellular permeability in Caco-2 cells; on the other hand, DSS suppressed MLCK expression in the colonic epithelium of DSS-colitis mice (Figure 6C) and increased the intestinal permeability (Figure 6A), which appears contradictory. Feighery et 


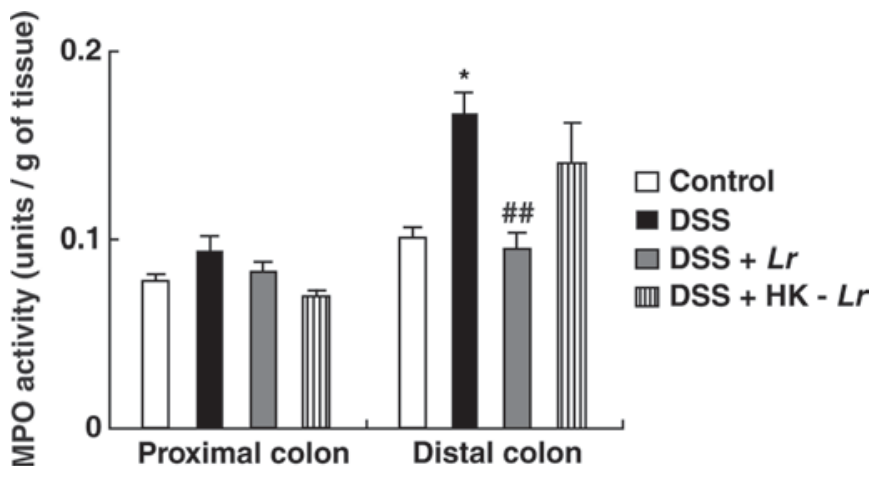

Figure 5. Effect of supplementation of live $(L r)$ or heat-killed (HKLr) Lactobacillus rhamnosus OLL2838 on myeloperoxidase (MPO) activity in the proximal and distal colon of dextran sodium sulfate (DSS)-induced colitis mice. On d 5, the mice were killed and the MPO activity was measured. Results are expressed as means \pm standard error $(\mathrm{n}=4) .{ }^{*} P<0.05$ versus control mice (none); \#\#P<0.01 versus DSS-treated mice (DSS).

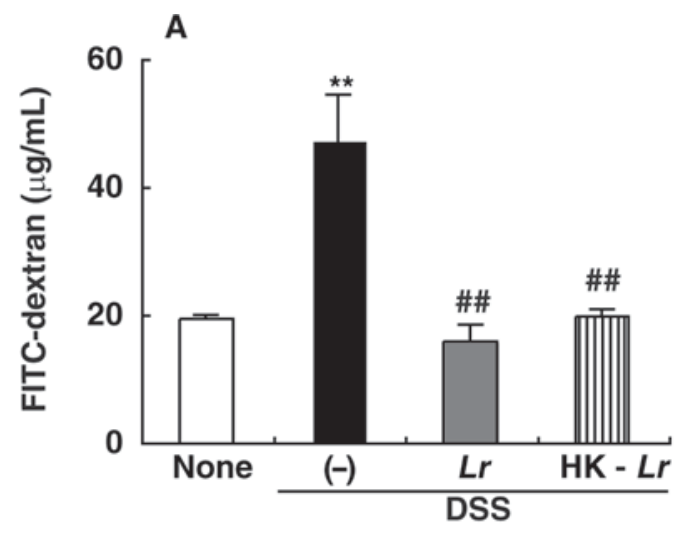

B
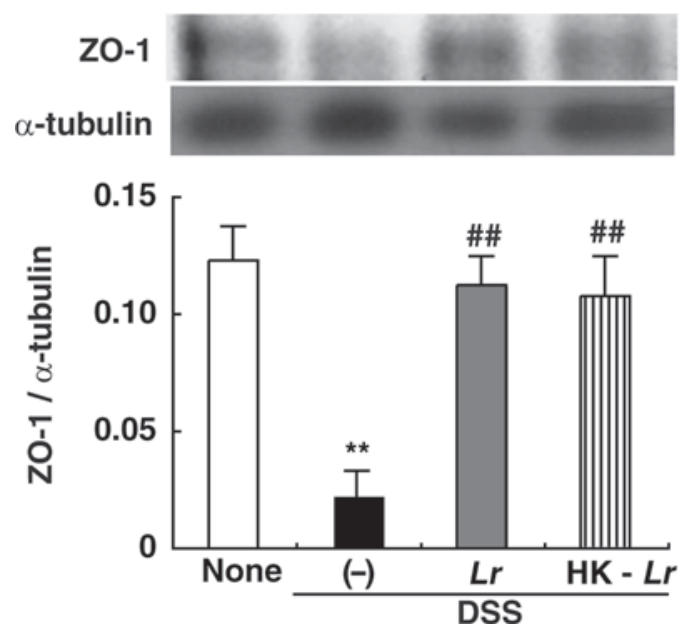

al. (2008) reported that the MLCK inhibitor reduces the increase in epithelial permeability in Caco-2 cells but not in DSS-induced colitis mice. Thus, the role of MLCK in intestinal permeability is probably different in Caco-2 cells and in DSS-induced colitis mice.

To our knowledge, there are few studies on the MLCK level in DSS-induced colitis mice. It has been reported that DSS induces apoptosis (Vetuschi et al., 2002; Cario et al., 2007) and that MLCK inhibition induces apoptosis (Fazal et al., 2005; Connell and Helfman, 2006); therefore, the intestinal epithelium of DSStreated mice with reduced MLCK expression (Figure 6C) may undergo apoptosis. This is the first finding that normalization of MLCK expression by OLL2838 can contribute to intestinal barrier recovery; OLL2838 probably suppressed apoptosis in the inflamed intestine.
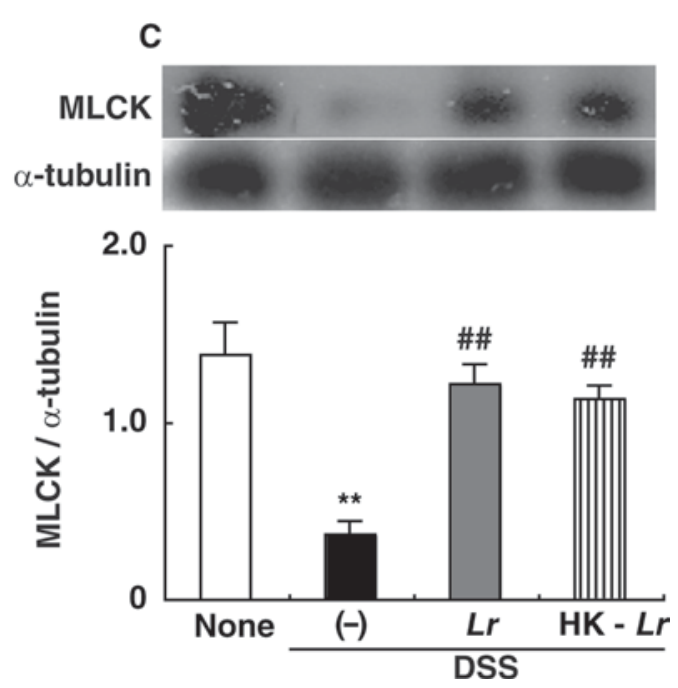

Figure 6. Protective effect of supplementation of live $(L r)$ or heat-killed (HK-Lr) Lactobacillus rhamnosus OLL2838 on increase in A) intestinal permeability and the expression of B) zonula occludens-1 (ZO-1) and C) myosin light-chain kinase (MLCK) on dextran sodium sulfate (DSS)-induced colitis mice. On d 5, mice $(\mathrm{n}=3)$ were gavaged with fluorescein isothiocyanate (FITC)-labeled dextran (60 mg/100 g of BW), and the serum level was measured $4 \mathrm{~h}$ later $(\mathrm{A})$. Separately, the proteins in the distal colons from each group $(\mathrm{n}=4)$ were extracted after the experiment shown in Figure 4 and used for SDS-PAGE and Western blotting. Images are representative of 3 independent experiments (B and C). Results are expressed as means \pm standard error. ${ }^{* *} P<0.01$ versus control mice (none); \#\#P $<0.01$ versus DSS-treated mice $(-)$. 
We demonstrated here that OLL2838 restored intestinal barrier function and paracellular permeability. In addition, mechanisms such as the suppression of proinflammatory cytokines in the intestine may exist for colitis prevention by OLL2838. We have recently reported that Bifidobacterium longum ssp. infantis suppressed proinflammatory IL-17 production in DSSinflamed colon organ culture (Tanabe et al., 2008). Lin et al. (2008) also reported that $L$. reuteri suppressed TNF- $\alpha$ and monocyte chemotactic protein-1 (MCP-1, one of the chemokines) in primary monocyte-derived macrophages from children with Crohn's disease, similar to that observed in the Caco-2 cells in this study (Figure 1B).

In conclusion, oral treatment with live and heat-killed OLL2838 suppressed colitis in mice. The OLL2838 strain prevented the loss of ZO-1 and MLCK and suppressed the increase in mucosal permeability. Studies in which probiotics were administered before DSS have indicated the preventive effects of probiotics in intestinal inflammation (Fitzpatrick et al., 2007; Chung et al. 2008). On the other hand, in this study, we administered DSS to mice before OLL2838 supplementation. By this method, we could determine whether OLL2838 prevents the development of colitis. Therefore, OLL2838 would be useful in the treatment of leaky gut and gastrointestinal diseases such as IBD.

\section{ACKNOWLEDGMENTS}

This study was partially supported by a Grant-in-Aid from the Japan Society for the Promotion of Science (No. 18658057).

\section{REFERENCES}

Cario, E., G. Gerken, and D. K. Podolsky. 2007. Toll-like receptor 2 controls mucosal inflammation by regulating epithelial barrier function. Gastroenterology 132:1359-1374.

Choi, C. H., T. I. Kim, S. K. Lee, K. M. Yang, and W. H. Kim. 2008. Effect of Lactobacillus GG and conditioned media on IL-1betainduced IL-8 production in Caco-2 cells. Scand. J. Gastroenterol. $6: 1-10$.

Chung, Y. W., J. H. Choi, T. Y. Oh, C. S. Eun, and D. S. Han. 2008. Lactobacillus casei prevents the development of dextran sulphate sodium-induced colitis in Toll-like receptor 4 mutant mice. Clin. Exp. Immunol. 151:182-189.

Connell, L. E., and D. M. Helfman. 2006. Myosin light chain kinase plays a role in the regulation of epithelial cell survival. J. Cell Sci. 119:2269-2281.

Fazal, F., L. Gu, I. Ihnatovych, Y. Han, W. Hu, N. Antic, F. Carreira, J. F. Blomquist, T. J. Hope, D. S. Ucker, and P. de Lanerolle. 2005. Inhibiting myosin light chain kinase induces apoptosis in vitro and in vivo. Mol. Cell. Biol. 25:6259-6266.

Feighery, L. M., S. W. Cochrane, T. Quinn, A. W. Baird, D. O’Toole, S. E. Owens, D. O'Donoghue, R. J. Mrsny, and D. J. Brayden. 2008. Myosin light chain kinase inhibition: correction of increased intestinal epithelial permeability in vitro. Pharm. Res. 25:13771386 .
Fitzpatrick, L. R., K. L. Hertzog, A. L. Quatse, W. A. Koltun, J. S. Small, and K. Vrana. 2007. Effects of the probiotic formulation VSL\#3 on colitis in weanling rats. J. Pediatr. Gastroenterol. Nutr. 44:561-570.

Gebska, A., R. Olszanecki, and R. Korbut. 2005. Endotoxaemia in rats: Role of leukocyte sequestration in rapid pulmonary nitric oxide synthase-2 expression. J. Physiol. Pharmacol. 56:299-311.

Gionchetti, P., F. Rizzello, C. Morselli, G. Poggiol, R. Tambasco, C. Calabrese, P. Brigidi, B. Vitali, G. Straforini, and M. Campieri. 2007. High-dose probiotics for the treatment of active pouchitis. Dis. Colon Rectum 50:2075-2082.

Kelly, D., J. I. Campbell, T. P. King, G. Grant, E. A. Jansson, A. G. Coutts, S. Pettersson, and S. Conway. 2004. Commensal anaerobic gut bacteria attenuate inflammation by regulating nuclearcytoplasmic shuttling of PPAR-gamma and RelA. Nat. Immunol. 5:104-112.

Krieglstein, C. F., C. Anthoni, W. H. Cerwinka, K. Y. Stokes, J. Russell, M. B. Grisham, and D. N. Granger. 2007. Role of bloodand tissue-associated inducible nitric-oxide synthase in colonic inflammation. Am. J. Pathol. 170:490-496.

Lee, J., J. H. Mo, K. Katakura, I. Alkalay, A. N. Rucker, Y. T. Liu, H. T. Lee, C. Shen, G. Cojocaru, S. Shenouda, M. Kagnoff, L. Eckmann, Y. Ben-Neriah, and E. Raz. 2006. Maintenance of colonic homeostasis by distinctive apical TLR9 signaling in intestinal epithelial cells. Nat. Cell Biol. 8:1327-1336.

Li, Q., Q. Zhang, M. Wang, S. Zhao, J. Ma, N. Luo, N. Li, Y. Li, $\mathrm{G}$. $\mathrm{Xu}$, and J. Li. 2008. Interferon-gamma and tumor necrosis factor-alpha disrupt epithelial barrier function by altering lipid composition in membrane microdomains of tight junction. Clin. Immunol. 126:67-80.

Lin, Y. P., C. H. Thibodeaux, J. A. Peña, G. D. Ferry, and J. Versalovic. 2008. Probiotic Lactobacillus reuteri suppress proinflammatory cytokines via c-Jun. Inflamm. Bowel Dis. 14:1068-1083.

Ma, T. Y., M. A. Boivin, D. Ye, A. Pedram, and H. M. Said. 2005. Mechanism of TNF- $\alpha$ modulation of Caco-2 intestinal epithelial tight junction barrier: role of myosin light-chain kinase protein expression. Am. J. Physiol. Gastrointest. Liver Physiol. 288:G422G430.

Ma, T. Y., G. K. Iwamoto, N. T. Hoa, V. Akotia, A. Pedram, M. A. Boivin, and H. M. Said. 2004. TNF-alpha-induced increase in intestinal epithelial tight junction permeability requires NFkappa B activation. Am. J. Physiol. Gastrointest. Liver Physiol. 286:G367-G376.

Mazzon, E., and S. Cuzzocrea. 2008. Role of TNF-alpha in ileum tight junction alteration in mouse model of restraint stress. Am. J. Physiol. Gastrointest. Liver Physiol. 294:G1268-G1280.

Meddings, J. 2008. The significance of the gut barrier in disease. Gut $57: 438-440$

Miyauchi, E., H. Morita, J. Okuda, T. Sashihara, M. Shimizu, and S. Tanabe. 2008. Cell wall fraction of Enterococcus hirae ameliorates TNF- $\alpha$-induced barrier impairment in the human epithelial tight junction. Lett. Appl. Microbiol. 46:469-476.

Poritz, L. S., K. I. Garver, C. Green, L. Fitzpatrick, F. Ruggiero, and W. A. Koltun. 2007. Loss of the tight junction protein ZO-1 in dextran sulfate sodium induced colitis. J. Surg. Res. 140:12-19.

Resta-Lenert, S., and K. E. Barrett. 2006. Probiotics and commensals reverse TNF- $\alpha$ - and IFN- $\gamma$-induced dysfunction in human intestinal epithelial cells. Gastroenterology 130:731-746.

Schlee, M., J. Harder, B. Köten, E. F. Stange, J. Wehkamp, and K. Fellermann. 2008. Probiotic lactobacilli and VSL\#3 induce enterocyte beta-defensin 2. Clin. Exp. Immunol. 151:528-535.

Seth, A., F. Yan, D. B. Polk, and R. K. Rao. 2008. Probiotics ameliorate the hydrogen peroxide-induced epithelial barrier disruption by a PKC- and MAP kinase-dependent mechanism. Am. J. Physiol. Gastrointest. Liver Physiol. 294:G1060-G1069.

Smits, H. H., A. Engering, D. van der Kleij, E. C. de Jong, K. Schipper, T. M. van Capel, B. A. Zaat, M. Yazdanbakhsh, E. A. Wierenga, Y. van Kooyk, and M. L. Kapsenberg. 2005. Selective probiotic bacteria induce IL-10-producing regulatory $\mathrm{T}$ cells in vitro by modulating dendritic cell function through dendritic cell-specific 
intercellular adhesion molecule 3-grabbing nonintegrin. J. Allergy Clin. Immunol. 115:1260-1267.

Soo, I., K. L. Madsen, Q. Tejpar, B. C. Sydora, R. Sherbaniuk, B. Cinque, L. Di Marzio, M. G. Cifone, C. Desimone, and R. N Fedorak. 2008. VSL\#3 probiotic upregulates intestinal mucosal alkaline sphingomyelinase and reduces inflammation. Can. J. Gastroenterol. 22:237-242.

Strober, W., I. Fuss, and P. Mannon. 2007. The fundamental basis of inflammatory bowel disease. J. Clin. Invest. 117:514-521.

Tanabe, S., Y. Kinuta, and Y. Saito. 2008. Bifidobacterium infantis suppresses proinflammatory interleukin-17 production in murine splenocytes and dextran sodium sulfate-induced intestinal inflammation . Int. J. Mol. Med. 22:181-185.

Vetuschi, A., G. Latella, R. Sferra, R. Caprilli, and E. Gaudio. 2002. Increased proliferation and apoptosis of colonic epithelial cells in dextran sulfate sodium-induced colitis in rats. Dig. Dis. Sci. 47:1447-1457.
Xavier, R. J., and D. K. Podolsky. 2007. Unravelling the pathogenesis of inflammatory bowel disease. Nature 448:427-434

Zeissig, S., N. Bürgel, D. Günzel, J. Richter, J. Mankertz, U. Wahnschaffe, A. J. Kroesen, M. Zeitz, M. Fromm, and J. D. Schulzke. 2007. Changes in expression and distribution of claudin 2,5 and 8 lead to discontinuous tight junctions and barrier dysfunction in active Crohn's disease. Gut 56:61-72.

Zhang, L., N. Li, R. Caicedo, and J. Neu. 2005. Alive and dead Lactobacillus rhamnosus GG decrease tumor necrosis factoralpha-induced interleukin- 8 production in Caco-2 cells. J. Nutr. 135:1752-1756

Zocco, M. A., L. Z. dal Verme, F. Cremonini, A. C. Piscaglia, E. C. Nista, M. Candelli, M. Novi, D. Rigante, I. A. Cazzato, V. Ojetti, A. Armuzzi, G. Gasbarrini, and A. Gasbarrini. 2006. Efficacy of Lactobacillus GG in maintaining remission of ulcerative colitis. Aliment. Pharmacol. Ther. 23:1567-1574. 